\title{
Procesamiento del grano de maíz sobre la cinética de degradación de la materia seca in vitro
}

\author{
Corn grain processing on the kinetics of in vitro dry matter \\ degradation
}

\author{
Raul Velásquez $\mathrm{V}^{1^{*}}$ M.Sc, Ricardo R Noguera, ${ }^{2}$ Ph.D, Sandra Posada $0,{ }^{2} \mathrm{Ph} . \mathrm{D}$.
}

\begin{abstract}
${ }^{1}$ Universidad Nacional de Colombia, Facultad de Ciencias Agrarias, Departamento de Producción animal. Medellín, Colombia. ${ }^{2}$ Universidad de Antioquia, Facultad de Ciencias Agrarias. Grupo de Investigación en Ciencias Agrarias - GRICA. Medellín, Colombia. ${ }^{*}$ Correspondencia: ravelasquezv@ unal.edu.co
\end{abstract}

Recibido: Abril de 2013; Aceptado: Agosto de 2013.

\section{RESUMEN}

Objetivo. Determinar el efecto del procesamiento del grano de maíz sobre la cinética de degradación y fermentación de la materia seca in vitro. Materiales y métodos. Se evaluaron cinco tratamientos, cada uno constó de una mezcla con dos especies, $70 \%$ pasto Kikuyo (Pennisetum clandestinum) y $30 \%$ grano de maíz (Zea mays). El grano fue sometido a diferentes procesamientos: maíz grueso seco (MGS), maíz fino seco (MFS), maíz reconstituido (MGH), reconstituido y ensilado (MGE) y ensilado con urea (MGEU). Se realizó la técnica de gas in vitro para medir parámetros de degradación y de producción de gas en diferentes horarios hasta las $48 \mathrm{~h}$. Se utilizaron los modelos de Gompertz y Ørskov y McDonald para ajustar las curvas de producción de gas y degradación de MS y se evaluaron con PROC NLIN de SAS. Resultados. Los mayores volúmenes acumulados fueron obtenidos con los tratamientos MFS y MGEU con $552.5 \mathrm{ml}$ y $524.03 \mathrm{ml}$, respectivamente y fueron diferentes al tratamiento MGS $(p<0.05)$. El potencial de degradación $(A+B)$ en MGS mostró el menor valor, siendo diferente $(p<0.05)$ a los tratamientos MFS, MGE y MGEU. Conclusiones. El tipo de procesamiento del grano de máiz molido fino, reconstituido, ensilado con y sin urea mejoraron significativamente los parámetros de fermentación y degradación de la MS. El uso de grano molido fino $(2 \mathrm{~mm})$ puede ser reemplazado por grano grueso reconstituido y ensilado, el cual resulta más económico que la molienda fina.

Palabras clave: Degradabilidad, in vitro, producción de gas, rumiantes (Fuente: MeSH).

\section{ABSTRAC}

Objective. Determine the effect of processing corn grain on the kinetics of degradation and in vitro fermentation of dry matter. Materials and methods. There were five treatments, each one consisting of a mixture of two species, $70 \%$ Kikuyu grass (Pennisetum clandestinum) and $30 \%$ corn (Zea mays). The grain was subjected to various procedures: dry coarse corn (DCC), fine dry corn (FDC), reconstituted corn (RC), reconstituted and ensiled (REC) and silage with urea (REUC). In vitro gas technique was used to measure degradation parameters and gas production at different times up to 
$48 \mathrm{~h}$. The Gompertz and the Ørskov and McDonald models were used to adjust gas production and degradation curves of DM, and they were evaluated with PROC NLIN from SAS. Results. The highest accumulated volumes were obtained from treatments with FDC and REUC with 524.03 and $552.5 \mathrm{ml}$, respectively and were different from the DCC treatment $(p<0.05)$. The potential of degradation $(A$ $+B)$ in DCC showed the lowest value being different $(p<0.05)$ with FDC, REC and REUC treatments. Conclusions. The type of processing of corn grain, as fine ground, reconstituted silage with and without urea, significantly improved the fermentation and degradation of DM parameters. The use of finely ground grain $(2 \mathrm{~mm})$ can be replaced by coarse reconstituted and silage, since it is cheaper than fine grinding.

Key words: Degradability, gas production, in vitro, ruminant (Source: MeSH).

\section{INTRODUCCIÓN}

El tipo de grano y su procesamiento influyen en la ganancia de peso de los animales a los que se les suministra como suplemento, asimismo, el objetivo del procesamiento es aumentar la disponibilidad de almidón y la densidad energética de la ración, además, el procesamiento puede destruir micotoxinas y mejorar las características de la dieta y con ello incrementar la respuesta productiva de animales (1). De otro lado, entre el 75 al $80 \%$ de los costos relacionados con la producción de ganado en confinamientos comerciales son de alimentación y los granos de estas dietas son procesados para mejorar la palatabilidad, modificar el tamaño de partícula, aumentar la digestibilidad, modificar la tasa, el sitio y la extensión de la digestión y facilitar su almacenamiento (2).

Uno de los granos de mayor importancia para suplementación animal es el maíz, ya que por sus características nutricionales y disponibilidad comercial es el alimento más utilizado en la formulación de raciones para animales, llegando a constituir más del $50 \%$ del volumen de la ración concentrada. Sin embargo el procesamiento del grano de maíz seco involucra gastos adicionales como transporte, secado y almacenamiento, sin contar que durante el proceso el grano almacenado puede servir de sustrato para el crecimiento de hongos e insectos, alterando su composición química y valor nutricional (3).

En este contexto, el ensilaje de granos húmedos puede ser una alternativa para su conservación y almacenamiento. El almacenamiento de los granos de maíz en la forma de ensilaje presenta ventajas económicas con relación a los granos secos, como la optimización del uso de la tierra, reducción de pérdidas en los periodos pre y post cosecha, economía en la mano de obra y costos de almacenamiento. Además, el ensilaje puede garantizar la calidad sanitaria del grano y mejorar la disponibilidad de nutrientes (4). El ensilaje de maíz desgranado de alta humedad o reconstituido se ha desarrollado como una alternativa viable y a veces se prefiere al maíz seco ya que posee un valor alimenticio igual al seco con base en la MS (5).

La reconstitución del grano se refiere a la adición de agua al grano maduro para aumentar su contenido de humedad de 25 a $30 \%$ y luego es almacenado en un silo durante un tiempo no menor 21 días (6). Otra manera de ensilar grano reconstituido es adicionándole urea, que tiene el potencial para preservar grano de sorgo de alta humedad y a la vez mejorar su calidad alimenticia; en el grano de maíz húmedo, la urea libera amoniaco que es tóxico para hongos, lo que evita la formación de toxinas nocivas para los animales (7)

El objetivo de este estudio fue determinar el efecto del procesamiento del grano de maíz sobre la cinética de degradación y fermentación de la materia seca in vitro.

\section{MATERIALES Y MÉTODOS}

Sitio de estudio. Este trabajo fue desarrollado en el Laboratorio NUTRILAB - GRICA, perteneciente a la Facultad de Ciencias Agrarias, ubicado en la Sede de Investigación Universitaria - Universidad de Antioquia, Medellín - Colombia.

Sustratos. Se utilizaron dos especies, una gramínea de clima frío Kikuyo (Pennisetum clandestinum) y grano entero de maíz (Zea mays). Al grano de maíz se le realizaron diferentes tipos de procesamiento los cuales diferenciaron los tratamientos: Maíz grueso seco (MGS): granos secos de maíz fueron molidos con un tamaño de partícula de $4 \mathrm{~mm}$; Maíz fino seco (MFS): granos secos de maíz fueron molidos con un tamaño de partícula de $1 \mathrm{~mm}$; Maíz grueso 
húmedo (MGH): granos de maíz con un tamaño de partícula de $4 \mathrm{~mm}$ fueron reconstituidos con agua hasta alcanzar un valor teórico de humedad de 20\%, este proceso duró 6 h; Maíz grueso ensilado (MGE): granos de maíz con un tamaño de partícula de $4 \mathrm{~mm}$ fueron reconstituidos hasta alcanzar un porcentaje de humedad del $25 \%$ y se ensilaron en microsilos de laboratorio por un periodo de 25 días; y Maíz grueso ensilado con urea (MGEU): el proceso fue igual al descrito para el procesamiento MGE, agregándole $2 \%$ de urea comercial con relación al peso de la masa ensilada.

Para los análisis todos los sustratos fueron molidos en un molino Thomas - Willey $®$, utilizando una criba de $1 \mathrm{~mm}$. Muestras de cada tratamiento fueron tomadas y analizadas en el laboratorio para determinar sus concentraciones de materia seca (MS) a $65^{\circ} \mathrm{C}$ durante $48 \mathrm{~h}$, proteína cruda (PC) (8), fibra detergente neutra (FDN) y fibra detergente ácida (FDA) por el método descrito por Van Soest et al (9), energía bruta mediante bomba calorimétrica, los valores de $\mathrm{pH}$ mediante potenciómetro (10) y nitrógeno amoniacal (NNH3) mediante el método de Kjeldahl (11). Los valores encontrados son mostrados en la tabla 1.

Tabla 1. Composición química de las materias primas del ensayo.

\begin{tabular}{|c|c|c|c|c|c|}
\hline \multirow{2}{*}{ Características } & \multicolumn{2}{|c|}{ Ensilaje } & \multirow{2}{*}{$\begin{array}{l}\text { Maíz } \\
\text { Seco }\end{array}$} & \multirow{2}{*}{$\begin{array}{c}\text { Maíz } \\
\text { húmedo }\end{array}$} & \multirow{2}{*}{ Kikuyo } \\
\hline & Maíz & $\begin{array}{c}\text { Maíz + } \\
\text { urea }\end{array}$ & & & \\
\hline MS\% & 58.31 & 60.52 & 87.00 & 83.21 & 17.3 \\
\hline $\begin{array}{l}\text { Proteína cruda, \% de } \\
\text { la MS }\end{array}$ & 8.19 & 11.26 & 8.71 & 8.70 & 12.99 \\
\hline $\begin{array}{l}\text { Energía Bruta, Mcal/ } \\
\text { KgMS }\end{array}$ & 4.36 & 4.42 & 4.32 & 4.32 & 4.04 \\
\hline $\mathrm{pH}$ & 4.38 & 4.44 & -- & -- & -- \\
\hline $\begin{array}{l}\text { Fibra en detergente } \\
\text { neutro, \% de la MS }\end{array}$ & 8.83 & 8.63 & 9.12 & 9.10 & 63.4 \\
\hline $\begin{array}{l}\text { Fibra en detergente } \\
\text { ácido, } \% \text { de la MS }\end{array}$ & 2.91 & 2.85 & 3.44 & 3.42 & 34.2 \\
\hline Nitrógeno Amoniacal, \% & 0.72 & 0.93 & -- & -- & -- \\
\hline
\end{tabular}

Tratamientos. La inclusión de las materias primas dentro de los tratamientos se hizo con base en la materia seca de los sustratos, de la siguiente manera:

MFS: $\quad$ Kikuyo $70 \%+30 \%$ maíz fino seco MGE: Kikuyo $70 \%+30 \%$ maíz grueso ensilado

MGEU: Kikuyo $68 \%+30 \%$ maíz grueso ensilado $+2 \%$ de urea

MGH: Kikuyo $70 \%+30 \%$ maíz grueso húmedo

MGS: Kikuyo $70 \%+30 \%$ maíz grueso seco

Preparación del medio. El día previo al inicio del ensayo se elaboró la solución tampón (12). La solución fue preparada con $9.80 \mathrm{~g} / \mathrm{L}$ de $\mathrm{NaHCO}_{3}$, $4.65 \mathrm{~g} / \mathrm{L}$ de $\mathrm{Na}_{2} \mathrm{HPO}_{4} 2 \mathrm{H}_{2} \mathrm{O}, 0.57 \mathrm{de} \mathrm{KCl}, 0.47 \mathrm{~g} / \mathrm{L}$ de $\mathrm{NaCL}, \quad 0.12 \mathrm{~g} / \mathrm{L} \mathrm{MgSO}_{4} .7 \mathrm{H}_{2} \mathrm{O}$ y $0.05 \mathrm{~g} / \mathrm{L}$ de $\mathrm{CaCl}_{2} \cdot 2 \mathrm{H}_{2} \mathrm{O}$ (13). Esta solución fue agitada con fuerza para permitir la mezcla completa de las soluciones y fue saturada con $\mathrm{CO}_{2}$ por dos $\mathrm{h}$, luego se almacenó a $39^{\circ} \mathrm{C}$.

Colecta de inóculo. La colecta de líquido ruminal se hizo de tres vacas Holstein fistuladas en el rumen, alimentadas con pasto kikuyo, a las 6:00 h. El líquido se retiró manualmente de diferentes partes del rumen y se almacenó en termos calentados a $40^{\circ} \mathrm{C}$ con agua. Luego, el líquido ruminal fue llevado a laboratorio y filtrado a través de paños de algodón, separando la parte líquida, que fue transferida a un erlenmeyer que se mantuvo a una temperatura de $39^{\circ} \mathrm{C}$ y saturada continuamente con $\mathrm{CO}_{2}$ para garantizar condiciones de anaerobiosis y la parte sólida, desechada.

\section{Preparación de los frascos de incubación.} Para determinar la producción de gas producto de la fermentación de MS se utilizó la técnica in vitro descrita por Mauricio et al (14) para la cual se usaron frascos de vidrio con capacidad de $100 \mathrm{ml}$. En cada uno de ellos fueron pesados aproximadamente $0.35 \mathrm{gr}$ de kikuyo y $0.15 \mathrm{~g}$ de los diferentes tipos de maíz o silo de maíz según el tratamiento. Adicionalmente a cada frasco se introdujo $5 \mathrm{ml}$ de líquido ruminal y $45 \mathrm{ml}$ de la solución tampón. Los frascos se sellaron con un tapón de caucho, se agitaron con la mano y se introdujeron en una estufa de ventilación forzada a $39^{\circ} \mathrm{C}$. El tiempo de inicio de incubación es llamado el tiempo cero.

Se utilizó, además, una serie de frascos (10) como blancos que contenían medio de cultivo e inóculo pero sin sustrato, para corregir la presión generada por la utilización de gas $\mathrm{CO}_{2}$ y la presión producida por la fermentación de los microorganismos presentes en el líquido ruminal (14).

Lecturas de producción de gas. La presión de gas se midió con un transductor digital de presión tipo OMEGA Modelo PX 605-030GI en cada uno de los frascos de incubación. Para ello, se acopló una aguja al transductor y se introdujo a través de la tapa de caucho de los frascos. La presión se midió en libras por pulgada cuadrada (PSI).

Las mediciones de presión de gas se hicieron a las $2,4,6,8,10,12,15,18,24,36$ y $48 \mathrm{~h}$ de incubación. Luego de cada medición se agitaron los frascos y fueron devueltos a incubación en la estufa de aire forzado. Para transformar los datos de presión a volumen se utilizó la ecuación $Y=-0.1375+5.1385 X+0.0777 X^{2}$ donde $Y$ representa el volumen de gas producido por cada unidad de presión (X) (15). 
Degradación in vitro de la MS. Para determinar la degradación de la MS a través del tiempo se retiraron 16 frascos del proceso de incubación (incluidos dos blancos) a las 6, 10, 15, 24 y 48 h. El residuo de cada incubación se filtró en papel filtro pesados con antelación, luego se secaron por $48 \mathrm{~h}$ a $65^{\circ} \mathrm{C}$ y luego se pesaron para determinar la MS desaparecida.

Factor de partición (FP). La producción de gas in vitro tiene una relación estrecha entre la producción de gas y la degradación de MS durante la fermentación. Esta relación sugiere que a mayor degradación de MS hay mayor producción de gas, lo cual no es cierto en todos los casos, ya que hay sustratos que presentan igual degradación de MS pero producen diferentes volúmenes de gas (15). Para dilucidar esto se calcula el parámetro factor de partición (FP), que relaciona la cantidad de sustrato degradado $(\mathrm{mg})$ y el volumen de gas producido $(\mathrm{ml})$. El FP se considera un factor de eficiencia microbiana (16).

Análisis estadístico. Con el fin de analizar el comportamiento de la fermentación y degradación en el tiempo, se utilizaron los modelos de Gompertz (17) y Ørskov y McDonald (18) para ajustar las curvas de producción de gas y degradación de MS, respectivamente, para ello fue utilizado el procedimiento NLIN de SAS (19). Asimismo, para analizar la influencia de los tratamientos en la degradación de la MS en el tiempo se realizó un análisis de medidas repetidas con el procedimiento PROC MIXED de SAS (19).

Para evaluar el efecto de los tratamientos sobre los parámetros de cinética de producción de gases y para comparar los tratamientos en los diferentes tiempos de incubación se utilizó la prueba de Tukey encontrándo diferencias con un $p<0.05$, con la sentencia PROC MIXED de SAS (19).

\section{RESULTADOS}

Producción de gas. En la tabla 2, se presentan los datos de producción acumulada de gas expresada en $\mathrm{ml} / \mathrm{g}$ de $\mathrm{MS}$ incubada hasta las $48 \mathrm{~h}$ de incubación para los cinco tratamientos.

Los valores de producción de gas para los cinco tratamientos no difieren estadísticamente hasta las $12 \mathrm{~h}$ de incubación $(p>0.05)$. Después de $15 \mathrm{~h}$ de incubación los tratamientos MGH y MGS produjeron un menor volumen de gas que los tratamientos con grano de MFS y MGEU $(p<0.05)$, indicando un efecto positivo del procesamiento de ensilado y molido sobre la
Tabla 2. Volumen de gas acumulado ( $\mathrm{ml} / \mathrm{gramo}$ de MS incubado) durante todos los horarios de medición para los tratamientos en estudio.

\begin{tabular}{cccccc}
\hline \multirow{2}{*}{$\begin{array}{c}\text { Tiempo } \\
\text { incubación }\end{array}$} & \multicolumn{5}{c}{ Tratamientos } \\
\cline { 2 - 6 } & MFS & MGE & MGEU & MGH & MGS \\
\hline 0 & $0.00^{\mathrm{a} *}$ & $0.00^{\mathrm{a}}$ & $0.00^{\mathrm{a}}$ & $0.00^{\mathrm{a}}$ & $0.00^{\mathrm{a}}$ \\
2 & $3.80^{\mathrm{a}}$ & $2.12^{\mathrm{a}}$ & $3.24^{\mathrm{a}}$ & $2.85^{\mathrm{a}}$ & $2.82^{\mathrm{a}}$ \\
4 & $5.90^{\mathrm{a}}$ & $3.62^{\mathrm{a}}$ & $5.13^{\mathrm{a}}$ & $4.30^{\mathrm{a}}$ & $4.71^{\mathrm{a}}$ \\
6 & $13.83^{\mathrm{a}}$ & $9.14^{\mathrm{a}}$ & $10.69^{\mathrm{a}}$ & $7.91^{\mathrm{a}}$ & $8.87^{\mathrm{a}}$ \\
8 & $27.30^{\mathrm{a}}$ & $20.13^{\mathrm{a}}$ & $21.70^{\mathrm{a}}$ & $13.14^{\mathrm{a}}$ & $14.94^{\mathrm{a}}$ \\
10 & $50.76^{\mathrm{a}}$ & $41.93^{\mathrm{a}}$ & $44.14^{\mathrm{a}}$ & $25.39^{\mathrm{a}}$ & $27.86^{\mathrm{a}}$ \\
12 & $76.63^{\mathrm{a}}$ & $63.39^{\mathrm{a}}$ & $70.39^{\mathrm{a}}$ & $40.33^{\mathrm{a}}$ & $41.02^{\mathrm{a}}$ \\
15 & $116.14^{\mathrm{a}}$ & $94.13^{\mathrm{ab}}$ & $108.09^{\mathrm{a}}$ & $63.85^{\mathrm{b}}$ & $63.82^{\mathrm{b}}$ \\
18 & $148.91^{\mathrm{a}}$ & $119.05^{\mathrm{ab}}$ & $138.71^{\mathrm{ab}}$ & $84.66^{\mathrm{b}}$ & $86.73^{\mathrm{b}}$ \\
24 & $206.43^{\mathrm{a}}$ & $164.16^{\mathrm{ab}}$ & $186.59^{\mathrm{ab}}$ & $124.06^{\mathrm{c}}$ & $135.18^{\mathrm{bc}}$ \\
36 & $314.19^{\mathrm{a}}$ & $230.90^{\mathrm{bc}}$ & $287.44^{\mathrm{ab}}$ & $174.41^{\mathrm{c}}$ & $211.51^{\mathrm{c}}$ \\
48 & $453.60^{\mathrm{a}}$ & $333.50^{\mathrm{b}}$ & $423.22^{\mathrm{a}}$ & $277.58^{\mathrm{b}}$ & $296.05^{\mathrm{b}}$ \\
\hline
\end{tabular}

*Letras diferentes en la misma fila indican valores que difieren estadísticamente $(p<0.05)$

producción de gas, esta tendencia se mantiene hasta las $48 \mathrm{~h}$ de incubación.

Parámetros estimados de producción de gas. En la tabla 3 se muestran los parámetros de la cinética de producción de gas para los cinco tratamientos estimados por el modelo de Gompertz. Los mayores volúmenes acumulados (VF) fueron obtenidos con los tratamientos MFS y MGEU con $552.5 \mathrm{ml}$ y $524.03 \mathrm{ml}$, respectivamente y fueron diferentes al tratamiento MGS $(p<0.05)$. Esto indica una producción similar de gas entre el alimento MFS y MGEU, mientras que no hubo diferencia $(p>0.05)$ entre los tratamientos MGE, MGH y MGS.

Tabla 3. Parámetros estimados de producción de gas ajustados al modelo de Gompertz para los tratamientos en estudio y Factor de partición a las $48 \mathrm{~h}$.

\begin{tabular}{llllll}
\hline \multirow{2}{*}{ Parámetros } & \multicolumn{5}{c}{ Tratamientos } \\
\cline { 2 - 6 } & \multicolumn{1}{c}{ MFS } & MGE & MGEU & MGH & MGS \\
\hline VF, ml & $552.50^{\mathrm{a}}$ & $381.53^{\mathrm{ab}}$ & $524.03^{\mathrm{a}}$ & $384.17^{\mathrm{ab}}$ & $236.85^{\mathrm{b}}$ \\
$\mathrm{L}, \mathrm{h}$. & $4.30^{\mathrm{b}}$ & $4.34^{\mathrm{b}}$ & $4.32^{\mathrm{b}}$ & $4.38^{\mathrm{b}}$ & $6.96^{\mathrm{a}}$ \\
$\mathrm{C}, \% \mathrm{~h}^{-1}$ & $0.06^{\mathrm{a}}$ & $0.07^{\mathrm{a}}$ & $0.06^{\mathrm{a}}$ & $0.06^{\mathrm{a}}$ & $0.011^{\mathrm{b}}$ \\
$\mathrm{FP}, \mathrm{mg}$ de MS/ & $0.66^{\mathrm{a}}$ & $0.91^{\mathrm{a}}$ & $0.71^{\mathrm{a}}$ & $0.96^{\mathrm{a}}$ & $0.82^{\mathrm{a}}$ \\
$\mathrm{ml} \mathrm{de} \mathrm{gas}$ & & & & &
\end{tabular}

$\mathrm{ml}$ de gas

$\mathrm{VF}=$ volumen acumulado de gas $(\mathrm{ml})$ correspondiente a la completa digestión del sustrato (asintota), L = tiempo de colonización( $\mathrm{h}$ ); C

= tasa constante de producción de gas del material potencialmente degradable $\left(\% \mathrm{~h}^{-1}\right) ; \mathrm{FP}=$ Factor de partición a las $48 \mathrm{~h}$. Letras diferentes en la misma fila difieren estadísticamente $(p<0.05)$.

El menor VF registrado en el tratamiento MGS está asociado con el tiempo de colonización (L), puesto que este tratamiento al tener un tamaño de partícula mayor y no sufrir un proceso de fermentación previo a través del ensilaje podría haber retardado la actividad microbiana y por ende los procesos de degradación y producción de gas. 
Asimismo, la tasa de producción de gas en el tratamiento MGS, presenta el menor valor luego de iniciado el proceso de degradación, mostrando diferencias significativas $(p<0.05)$ con respecto al resto de tratamientos. Por otro lado el factor de partición no presentó diferencias entre tratamientos, indicando que no fue afectado por el tipo de procesamiento del grano.

Parámetros de degradación de la MS. Los parámetros de degradación estimados por el modelo propuesto por Ørskov y McDonald (18) son presentados en la tabla 4.

Tabla 4. Valores promedio de los parámetros de degradación estimados a partir de la técnica in vitro de producción de gas.

\begin{tabular}{|c|c|c|c|c|c|}
\hline \multirow{2}{*}{ Parámetros } & \multicolumn{5}{|c|}{ Tratamientos } \\
\hline & MFS & MGE & MGEU & MGH & MGS \\
\hline$A$ & $9.30^{a *}$ & $3.48^{b}$ & $9.237^{a}$ & $11.46^{\mathrm{a}}$ & $3.29^{b}$ \\
\hline B & $57.42^{\mathrm{ab}}$ & $65.12^{\mathrm{a}}$ & $69.27^{a}$ & $48.24^{\mathrm{bc}}$ & $40.45^{c}$ \\
\hline C & $0.05^{\mathrm{b}}$ & $0.04^{b}$ & $0.03^{b}$ & $0.05^{b}$ & $0.10^{\mathrm{a}}$ \\
\hline $\begin{array}{l}\text { Potencial de } \\
\text { degradación } \\
(A+B)\end{array}$ & $66.72^{\mathrm{ab}}$ & $68.60^{\mathrm{ab}}$ & $78.51^{\mathrm{a}}$ & $59.69^{\mathrm{bc}}$ & $43.74^{c}$ \\
\hline $\begin{array}{l}\text { Fracción indigestible } \\
\text { (FI) }\end{array}$ & $33.27^{b c}$ & $31.39^{b c}$ & $21.49^{c}$ & $40.31^{\mathrm{ab}}$ & $56.25^{\mathrm{a}}$ \\
\hline $\begin{array}{l}\text { Degradación efectiva } \\
\text { (DE) }\end{array}$ & $37.59^{\mathrm{a}}$ & $32.10^{\mathrm{bc}}$ & $34.62^{\mathrm{ab}}$ & $34.69^{\mathrm{ab}}$ & $29.13^{c}$ \\
\hline
\end{tabular}
(DE)

*Letras distintas en una misma línea indican valores estadísticamente diferentes $(p<0.05), A$ : fracción rápidamente degradable, B: fracción de lenta degradación, $C$ : tasa constante de degradación de la fracción $B, A+B$ : potencial de degradación, fracción indigestible $=100-(A+B)$, Degradación efectiva: Calculada con una tasa de pasaje teórica de $5 \%$ por hora.

El mayor valor para la fracción rápidamente degradable (A) lo obtuvo el tratamiento $\mathrm{MGH}$, siendo diferente $(p<0.05)$ a los valores de MGS y $M G E$, quienes obtuvieron valores 8 puntos porcentuales menos en promedio.

Por otro lado, la fracción B se comportó de manera diferente, mostrando los mayores valores para los tratamientos MGE, MGEU y MFS. Este hecho indica que el procesamiento de ensilaje con y sin urea y el molido fino del grano de maíz mejora la digestibilidad de la fracción de lenta degradación con respecto al grano grueso húmedo y seco (MGS y MGH).

El tratamiento MGS mostró los menores valores para los parámetros $A$ y $B$, siendo diferentes $(p<0.05)$ a los demás tratamientos. Pero al comparar la velocidad de degradación de la fraccion $B$ de éste tratamiento, se observa una superioridad frente a los demás tratamientos.

El potencial de degradación $(A+B)$ en MGS mostró el menor valor, siendo diferente $(p<0.05)$ a los tratamientos MFS, MGE y MGEU, así el procesamiento del grano de maíz molido fino, reconstituido, ensilado y adicionándole urea tiene más ventajas degradativas que el grano grueso y seco. Por el contrario, la fracción indigestible fue mayor $(p<0.05)$ en el tratamiento MGS que en los tratamientos MFS, MGE y MGEU.

Considerando una tasa de pasaje del $5 \% / \mathrm{h}$ el valor de la DE fue menor en el tratamiento MGS con $29.13 \%$. Este valor fue significativamente inferior a los encontrados para los tratamientos MFS, MGEU y MGH, cuyos valores fueron $37.59,34.62$ y $34.69 \%$ respectivamente. Aparentemente, el molido fino, la reconstitución y el proceso de ensilaje con urea mejoran la degradabilidad del grano.

Los porcentajes de degradación in vitro de la MS de los cinco tratamientos en los diferentes horarios son presentados en la tabla 5 .

Tabla 5. Porcentaje de degradación de la materia seca de los tratamientos en diferentes horarios de incubación in vitro.

\begin{tabular}{cccccc}
\hline \multirow{2}{*}{ Horario } & \multicolumn{5}{c}{ Tratamientos } \\
\cline { 2 - 6 } & MFS & MGE & MGEU & MGH & MGS \\
\hline 6 & $24.05^{\mathrm{a}}$ & $13.64^{\mathrm{c}}$ & $15.8^{\mathrm{c}}$ & $22.54^{\mathrm{ab}}$ & $18.02^{\mathrm{bc}}$ \\
10 & $30.8^{\mathrm{a}}$ & $18.71^{\mathrm{c}}$ & $21.69^{\mathrm{bc}}$ & $23.28^{\mathrm{bc}}$ & $25.54^{\mathrm{b}}$ \\
15 & $36.26^{\mathrm{ab}}$ & $37.46^{\mathrm{ab}}$ & $38.05^{\mathrm{ab}}$ & $39.28^{\mathrm{a}}$ & $34.02^{\mathrm{b}}$ \\
24 & $52.25^{\mathrm{a}}$ & $43.76^{\mathrm{b}}$ & $44.78^{\mathrm{b}}$ & $45.94^{\mathrm{b}}$ & $39.69^{\mathrm{b}}$ \\
48 & $59.95^{\mathrm{a}}$ & $57.79^{\mathrm{a}}$ & $59.86^{\mathrm{a}}$ & $52.5^{\mathrm{b}}$ & $42.14^{\mathrm{c}}$ \\
\hline
\end{tabular}

*Letras diferentes en la misma fila difieren estadísticamente $\quad(p<0.05)$

En las primeras $6 \mathrm{~h}$ el porcentaje de degradación fue superior $(p<0.05)$ para los tratamientos MFS y $M G H$, mostrando un efecto positivo del molido fino y de la reconstitución, sobre los tratamientos ensilados y el maíz grueso seco. Entre las 10 y $15 \mathrm{~h}$ de incubación se observa que el MGH mostro el mayor porcentaje de degradación, siendo diferente únicamente a MGS, mostrando el efecto positivo del procesamiento de reconstitución del grano de maíz. Luego, en el horario entre las 24 y las $48 \mathrm{~h}$ de incubación se observa que la mayor extensión de la degradación de la MS fue observada en los tratamientos MFS, MGEU y MGE quienes fueron similares entre si $(p>0.05)$, pero presentaron diferencias significativas $(p<0.05)$ con los tratamientos MGH y MGS, indicando la favorabilidad de los tipos de procesamiento molido fino, ensilaje con y sin urea del grano de maíz sobre la degradabilidad de la MS.

\section{DISCUSIóN}

Producción de gas in vitro. La mayor producción de gas de los tratamientos MFS, MGE y MGEU (Tablas 2 y 3 ) puede deberse, primero, a que el maíz molido fino tiene una superficie 
de contacto mucho mayor que el maíz grueso y por tanto, los microorganismos ruminales tendrían una mayor posibilidad de atacar las moléculas de almidón del maíz, fermentando más rápidamente este tipo de carbohidratos y en consecuencia produciendo mayores volúmenes de gas. En el maíz grueso ensilado con y sin urea, el efecto de la reconstitución del grano y su posterior ensilado permiten la solubilización de la matriz proteica que rodea los gránulos de almidón del endospermo (20), hecho que permitiría una más rápida colonización y fermentación por parte de los microorganismos ruminales con la consecuente mayor producción de gas. Adicionalmente, la utilización de urea en el ensilaje produce amoniaco y éste a su vez provoca un ablandamiento del pericarpio del grano, aumentando la posibilidad de fermentación del mismo (21).

Resultados similares son reportados por Lara et al (22) quienes evaluando el efecto de la adición de maíz molido al follaje de Morera (Morus alba) sobre la degradabilidad y cinética de producción de gas in vitro, encontraron un efecto positivo sobre la degradación de MS y una mayor producción de gas en los tratamientos que incluyeron maíz molido en comparación con aquellos que solo incluían forraje de Morera. Asimismo, Noguera et al (23) reportaron un aumento significativo de la producción de gas in vitro al adicionar harina de papa (Solanum tuberosum) (carbohidratos solubles) al pasto kikuyo (Pennisetum clandestinum) que al compararlo con la producción de gas in vitro del pasto solo. Por su parte, Estévez et al (24) manifiestan que este efecto se puede deber a una mayor concentración de materia orgánica fácilmente degradable, promoviendo el desarrollo de las poblaciones microbianas ruminales y la fermentación. Asimismo, Noguera et al (23) menciona que al aumentar la disponibilidad de los carbohidratos no estructurales de rápida degradación en la dieta se incrementa el volumen final de producción de gas.

El volumen de gas producido y la degradación de la MS presentan una correlación positiva que implica que al aumentar la degradación de MS, aumenta la producción de gas. Las mayores producciones de gas y los mayores porcentajes de degradabilidad de la MS se dieron en los tratamientos MFS, MGE y MGEU, lo que indica que el grado de procesamiento del grano (molido fino, reconstituido, ensilado con y sin urea) aumenta la degradación de MS y la producción de gas.

Parámetros estimados de producción de gas in vitro. Los volúmenes de gas variaron de acuerdo con el tipo de procesamiento del grano, por lo cual se observó mayor volumen final de gas con los tratamientos MFS y MGEU (Tablas 2 y 3), indicando que los valores aumentan en los tratamientos que incluyeron la molienda fina y el ensilaje de grano reconstituido con urea, mientras que con el grano grueso seco, reconstituido y ensilado son menores. Esto se debe posiblemente a la disminución del tamaño de partícula que incrementa el área de exposición de los gránulos de almidón al ataque enzimático de los microorganismos ruminales. La acción mecánica del procesamiento de molido, reconstituido y ensilado produce cierto grado de gelatinización, en la cual los gránulos de almidón incrementan absorción de agua, expandiéndose y liberando parte de la amilosa, aumentando su susceptibilidad a la hidrólisis enzimática y su velocidad de fermentación.

El proceso de gelatinización comienza con el rompimiento de los enlaces con la amilasa en la zona amorfa del almidón, mientras que la presencia de agua y calor en la región cristalina sucede mas lentamente, facilitada por la mayor plasticidad de la región amorfa. De ahí que en el proceso de reconstitución del grano y la presencia de agua aumenta la plasticidad de las regiones amorfas incrementando la desestructuración de las regiones cristalinas (25).

El tiempo de colonización también tiene una relación directa con el tamaño de partícula y con el tipo de procesamiento. Se presenta un menor tiempo de colonización en los tratamientos donde el grano ha sido molido fino, o ha sido reconstituido, y/o ha sido ensilado. Noguera et al (23) aseguran que el metabolismo de los microorganismos ruminales se regula por la cantidad de carbohidratos no estructurales (CNE) en la ración y el tipo de procesamiento al cual hayan sido sometidos (químicos o físicos), ya que al aumentar la densidad energética con CNE procesados se promueve un rápido crecimiento microbiano, y se aumenta también la producción de ácidos grasos volátiles y así el volumen final de gas.

Los valores de la tasa de producción de gas (C) son proporcionales a la cantidad de CNE degradados, el sustrato con mayor tasa de degradación se asocia a mayor hidratación, concentración y contacto físico con microorganismos ruminales (26). Así el valor de C en el tratamiento MGS indica que su estructura presenta barreras físicas que evitan su hidrólisis, lo cual puede explicarse por su tamaño de partícula y que no sufrió ningún procesamiento a priori que promoviera la gelatinización y/o solubilidad del gránulo de almidón, dificultando su fermentación. 
Parámetros de degradación de la MS in vitro. La proporción de constituyentes solubles (fracción A) y de lenta degradación (B) presentes en el grano están relacionados con el tipo de procesamiento del grano. La molienda fina y la reconstitución del grano incrementaron la disponibilidad de los $\mathrm{CNE}$, permitiendo a los microorganismos colonizar rápidamente el sustrato e iniciar el proceso de degradación y fermentación (Tabla 4). El potencial de degradación $(A+B)$ de la MS es mayor para los tratamientos donde el grano ha sido molido, reconstituido, ensilado con o sin urea. Estos tipos de procesamiento dan mayor accequibilidad a los microorganismos para tener un contacto físico más eficiente con los gránulos de almidón y de ellos obtienen energía suficiente para su crecimiento y así pueden aumentar la degradación de los carbohidratos estructurales de la pared celular.

El proceso de reconstitución y ensilado mejora la degradabilidad ruminal del grano de maíz (27), coincidiendo con lo encontrado en este ensayo, donde los tratamientos con procesos de molido fino y ensilados presentaron mayor $(p<0.05)$ degradabilidad que el tratamiento seco y el reconstituido sin ensilar. En el proceso de reconstitución del grano y posterior ensilado, el agua aumenta la plasticidad de las regiones amorfas del almidón favoreciendo en gran medida la pérdida de estructura de las regiones cristalinas del mismo. La gelatinización comienza por la ruptura de enlaces con amilosa en la zona amorfa del almidón, mientras que la penetración de calor y agua en la región cristalina ocurre más lentamente, ayudada por la mayor plasticidad de la región amorfa (25).

De hecho, si se somete el almidón a reconstitución y a calentamiento se incrementa su gelatinización, lográndose una considerable degradación enzimática (28). Singh et al (29) haciendo una evaluación al interior del silo de grano de maíz desde en el inicio del proceso de ensilaje, mencionan que el almidón de los granos sufre gelatinización y en este proceso, ocurre la ruptura de los puentes de hidrógeno más débiles, que se unen a las cadenas de amilasa y amilopectina. La gelatinización involucra cambios irreversibles, hinchando y disrumpiendo el gránulo, perdiendo así su cristalinidad. Durante éste proceso las moléculas de almidón vibran rompiendo puentes de hidrógeno intermoleculares de las zonas amorfas de los gránulos, hidratándose hasta hincharse irreversiblemente, ligándose a la estructura finalmente (30), permitiendo así un mayor contacto físico de los microorganismos y facilitando la fermentación y degradación de la MS.

Posiblemente la gelatinización puede ocurrir por la presencia de los ácidos dentro del ensilaje, generando mayor degradación de MS durante la fermentación ruminal. También puede suceder, por un lado, que el mayor contenido de humedad de los granos favorece la fermentación y elevación de temperatura en el silo, causando una gelatinización parcial del almidón y aumentando su digestibilidad ruminal e intestinal; por otro, ocurre un efecto de solubilización de la matriz proteica alrededor de los gránulos de almidón facilitando el ataque enzimático de las bacterias ruminales (29).

La degradación efectiva considerando una tasa de pasaje del $5 \%$ por hora, presenta el mismo comportamiento que el potencial de degradación, indicando que los tratamientos que presentan grano molido fino, reconstituido y ensilado con úrea mejoran la disponibilidad energética de la ración mejorando los parámetros de degradación de la MS. La degradación efectiva fue mayor en los tratamientos que presentaron mayor potencial de degradación (Tabla 4). De hecho, en los tratamientos con mayor fermentación, hubo mayor actividad enzimática sobre el almidón. En el caso del tratamiento MGEU, una mayor disponibilidad de nitrógeno aportado por la urea, habría favorecido el crecimiento microbiano y por tanto la degradación de MS. Los tratamientos que presentaron procesamiento de molido, reconstituido y ensilado, ofrecieron un mayor aporte energético fermentable lo que incrementa la degradación efectiva de la ración, incrementando la síntesis de proteína microbiana $(1,28,29)$. Un resultado similar encontraron Svihus et al (28) quienes evaluaron el efecto del hojuelado al vapor sobre la degradabilidad ruminal del grano de maíz, encontrando un aumento en la degradabilidad efectiva del grano.

En conclusión el tipo de procesamiento del grano de máiz molido fino, reconstituido, ensilado con y sin urea mejoraron significativamente los parámetros de fermentación y degradación de la MS. Los mejores parámetros de degradación fueron para los tratamientos MFS, MGE y MGEU, lo que indica que uso de grano molido fino $(2 \mathrm{~mm})$ puede ser reemplazado por grano quebrado reconstituido y ensilado.

\section{Agradecimientos}

Al Ministerio de Agricultura y Desarrollo Rural de la República de Colombia, a COLANTA y a la estrategia de sostenibilidad 2011 - 2012 de la Vicerectoria de Investigaciones de la Universidad de Antioquia por la financiación de este trabajo. Este artículo hace parte del proyecto de grado de doctorado en Ciencias Animales de la Universidad de Antioquia del primer autor. 


\section{REFERENCIAS}

1. Klopfenstein TJ, Erickson GE, Bremer VR. Board-Invited Review: Use of distillers byproducts in the beef cattle feeding industry. J Anim Sci 2008; 86(5):1223-31.

2. Richards CJ, Hicks B. Processing of corn and sorghum for feedlot cattle. Vet Clin North Am Food Anim Pract 2007; 23(2):207-21.

3. Henrique $\mathrm{W}$, Beltrame JA, Leme $\mathrm{PR}$, Lanna D, Alleoni G, Coutinho Filho Jl, Sampaio A. Avaliação da silagem de grãos de milho úmido com diferentes volumosos para tourinhos em terminação: Desempenho e características de carcaça. R Bras Zootec 2007; 36(1):183-90.

4. Jobim CvC, Nussio LG, Reis RA, Schmidt P. Avances metodologicos na avaliação da qualidade da forragem conservada. R Bras Zootec 2007; 36(suppl):101-119.

5. Lohmann AC, Pozza PC, Nunes RV, Pozza MSS, Venturi I, Pasquetti TJ. Digestibilidade da silagem de grãos úmidos de milho com diferentes granulometrias para suínos. Arq Bras Med Vet Zootec 2010; 62(1):154-62.

6. Abdelhadi LO, Santini FJ, Gagliostro GA. Corn silage or high moisture corn supplements for beef heifers grazing temperate pastures: effects on performance, ruminal fermentation and in situ pasture digestion. A Feed Sci and Tech 2005; 118(1):63-78.

7. Eastridge ML. Major advances in applied dairy cattle nutrition. J Dairy Sci 2006; 89(4):131123.

8. AOAC International. Official methods of analysis. 17 Ed. Gaithersburg: AOAC International. 2003.

9. Van Soest PJ, Robertson JB, Lewis BA. Methods for dietary fiber, neutral detergent fiber, and nonstarch polysaccharides in relation to animal nutrition. J Dairy Sci 1991; 74(10):3583-3597.

10. Galina MA, Delgado-Pertiñez M, Ortíz-Rubio MA, Pineda LJ, Puga DC. Cinética ruminal y crecimiento de cabritos suplementados con un probiótico de bacterias ácido-lácticas. Revista Pastos y Forrajes 2009; 32(4):1-1.
11. B.S.I British Standards Institution. British Standard 696. Gerber Method for determination of fat in milk and milk products. London: British Standards Institution. 1955.

12. McDougall EI. Studies on ruminant saliva. 1. The composition and output of sheeps saliva. Biochem J 1948; 43:99-109.

13. Silva DJ. Análise de alimentos. Métodos químicos e biológicos. Minas Gerais: Universidad Federal de Viçosa. 1990.

14. Mauricio RM, Mould FL, Dhanoa MS, Owen E, Channa KS, Theodorou MK. A semi-automated in vitro gas production technique for ruminant feedstuff evaluation. A Feed Sci and Tech 1999; 79:320-330.

15. Posada SL y Noguera RR. Técnica in vitro de producción de gases: Una herramienta para la evaluación de alimentos para rumiantes. Lives Res Rural Develop. 2005; 17(4). URL Disponible en: http://www.Irrd.org/Irrd17/4/ posa17036.htm

16. Duque M, Noguera RR, y Restrepo LF. Efecto de la adición de urea protegida y sin protección sobre la cinética de degradación in vitro del pasto estrella (Cynodon nlemfluensis) y caña de azúcar (Saccharum officinarum). Lives Res Rural Develop 2009; 21(4). URL Disponible en: http://www.Irrd.org/Irrd21/4/duqu21058. htm

17. Lavrenčič A, Stefanon B, Susmel P. An evaluation of the Gompertz model in degradability studies of forage chemical components. Anim Sci 1997; 64:423-31.

18. Ørskov ER, McDonald I. The estimation of protein degradability in the rumen from incubation measurements weighted according to rate of passage. J Agric Sci Cambridge 1979; 92:499-503.

19. SAS/STAT: Guide for Personal Computer Versión 8.2. Cary (NC): SAS Institute Incorporation; 2001.

20. Corona L, Owens FN, Zinn RA. Impact of corn vitreousness and processing on site and extent of digestion by feedlot cattle. J Anim Sci 2006; 84(11):3020-31. 
21. Wood KM, Salim H, McEwen PL, Mandell IB, Miller SP, Swanson KC. The effect of corn or sorghum dried distillers grains plus solubles on growth performance and carcass characteristics of cross-bred beef steers. A Feed Sci and Techn 2011; 165(1):23-30.

22. Lara PE, Canché MC, Magaña H, Aguilar E, Sanginés JR. Producción de gas in vitro y cinética de degradación de harina de forraje de morera (Morus alba) mezclada con maíz. R Cub Cienc Agríc 2009; 43(3):273-279.

23. Noguera RR, Ramírez IC, Bolivar DM. Efecto de la inclusión de papa (Solanum tuberosum) en la cinética de fermentación in vitro del pasto kikuyo (Pennisetum clandestinum). Lives Res Rural Develop 2006; 18(5). URL Disponible en: http://www.Irrd.org/Irrd18/5/ nogu18062.htm

24. Estévez, OV, Pedraza RM, Guevara RV, Parra CE. Composición química y degradabilidad ruminal del follaje de tres variedades de Polyscias guilfoylei en la época de seca. Revista Pastos Forrajes 2004; 27(2):177-181.

25. Andrade H, García T, Kawas, J. Ruminal fermentation modification of protein and carbohydrate by means of roasted and estimation of microbial protein synthesis. R Bras Zootec 2009; 38(SPE):277-291.
26. Almaraz I, Losada H, Cortés J, Vargas J, Miranda L y Sánchez J. Producción de gas in vitro de desechos de verduras usados para alimentar vacas lecheras. Lives Res Rural Develop 2012; 24(8). URL Disponible en: http://www.Irrd.org/Irrd24/8/alma24132.htm

27. Almeida JG, Costa C, Monteiro AL, Garcia CA, Munari DP, Neres MA. Desempenho, características de carcaça e resultado econômico de cordeiros criados em creep feeding com silagem de grãos úmidos de milho. R Bras Zootec 2004; 33(4):1048-1059.

28. Mahasukhonthachat K, Sopade PA, Gidley MJ. Kinetics of starch digestion in sorghum as affected by particle size. J Food Engin 2010; 96(1):18-28.

29. Singh J, Dartois A, Kaur L. Starch digestibility in food matrix: a review. Trends Food Sci Tech 2010; 21(4):168-180.

30. Svihus B, Anne KU, Odd MH. Effect of starch granule structure, associated components and processing on nutritive value of cereal starch: A review. Anim Feed Sci Tech 2005; 122(3):303-320. 